\title{
Die Arbeitsgruppe Qualität AGQ-FMH sucht neue Mitglieder
}

\section{Martina Hersperger, \\ Leiterin Abteilung DDQ}

Peltenburg M, Kernen H, Schneider P, von Below GC, Waldis $\mathrm{G}$ et al. Qualität - ein Zusammenspiel aller Kräfte im ärztlichen Umfeld. Schweiz Ärztezeitung. 2005;86(18):1055-8.
Die Arbeitsgruppe Qualität ist seit 2005 eine beratende Kommission des Zentralvorstands. Sie umfasst eine Steuergruppe und einen Expertenpool und steht unter der Leitung von Olivier Kappeler, ZV-Mitglied Ressort DDQ (Daten, Demographie und Qualität). Die Steuergruppe besteht aus sechs bis acht FMH-Mitgliedern (bisher zwei aus der Romandie, vier aus der Deutschschweiz) mit beruflicher Tätigkeit im ambulanten oder stationären Sektor. Für den Expertenpool konnten ein internationaler Vertreter sowie je ein Vertreter der Hochschulen und der Kostenträger gewonnen werden. Die Abteilung DDQ leistet Unterstützungs- und Koordinationsarbeit.

\section{Aufgaben der AGQ}

- Das Leitbild «Ärztliche Qualität»* weiterentwickeln und umsetzen

- Impulse zur Begriffsklärung und zur Qualitätsentwicklung innerhalb der Ärzteschaft erarbeiten und umsetzen

- Grundlagen für das Zusammenspiel von Regulation, nachgewiesenen Fähigkeiten und Resultaten erarbeiten

- Stellungnahmen und Positionspapiere zu Händen des Zentralvorstands FMH erarbeiten

- Auf der Grundlage des Qualitätsverständnisses der Schweizer Ärzteschaft mit den Ansprechpartnern in Verhandlungen treten

Nach dem Rücktritt eines französischsprechenden Vertreters sucht die AGQ für die Steuergruppe 1-2 neue Mitglieder, vorzugsweise aus der Westschweiz oder dem Tessin.

\section{Aufgaben der Steuergruppe AGQ}

- Steuern des Gesamtprogramms

- Sichern der Fachlichkeit bzgl. Qualität (gemeinsam mit Expertenpool)

- Einspeisen von Trends und neuesten Entwicklungen

- Abgeben von Stellungnahmen zu Fachinhalten und neuen Entwicklungen

- Sichern des Anspruchsgruppen-Managements

- Möglichkeit zur Mitarbeit in Projekten

\section{Anforderungen, Aufwand}

\section{und Entschädigung}

- Interesse an und Erfahrung in der ärztlichen Qualität

- Sprachverständnis Deutsch und Französisch

- FMH-Mitglied

- 6 Sitzungen pro Jahr $(5 \times$ halber Tag, $1 \times$ ganzer Tag) inkl. Vor- und Nachbereitung

- Sitzungsort Bern (in der Regel)

- Entschädigung gemäss Spesenreglement FMH

- Bei Projektmitarbeit individuelle Entschädigungsregelung

\section{Interessiert?}

Es besteht die Möglichkeit, als Gast an einer Sitzung teilzunehmen. Die folgenden Mitglieder der Steuergruppe erteilen gerne Auskunft und freuen sich über die Kontaktaufnahme Interessierter:

Olivier Kappeler, Zentralvorstandsmitglied Ressort DDQ: olivier.kappeler@hin.ch

Patrick Bovier, Westschweizer Mitglied Steuergruppe AGQ: patrick.bovier@hcuge.ch Martina Hersperger, Leiterin Abteilung DDQ: martina.hersperger@fmh.ch 